\title{
Stoichiometry Fitter, a GUI for Fitting Solid Solutions and Analyzing Mineral Phases
}

\section{Z. Gainsforth ${ }^{1}$}

1. Space Sciences Laboratory, University of California Berkeley, 7 Gauss Way, Berkeley, CA, 94720

We have developed a software tool for analysis of multiphase quantification of materials characterized by techniques such as X-ray fluorescence (XRF) in a synchrotron and energy dispersive spectroscopy (EDS) in a transmission electron microscope (TEM). Quantifications from various techniques can be input as atomic $\%$, elemental weight $\%$, oxide weight $\%$ (with cation oxidation states) or as raw counts in the case of TEM measurements. In the latter case it is possible to apply a set of k-factors, a thickness correction and a detector correction. The thickness correction is based on scattering factors from the Center for X-ray Optics (CXRO) [1,2] and is found to be surprisingly accurate. On a troilite FIB section which was FIBed to $500 \mathrm{~nm}$ thickness, the optimal thickness correction resulting in perfect stoichiometry between Fe and S was $508 \mathrm{~nm}$. Generally, CXRO tabulated absorption values are accurate better than $10 \%$. The detector correction includes the Si dead layer, conductor coating, thin window, and finally accounts for incomplete absorption of photons at higher energies that pass through the detector entirely.

Regardless of how the elemental quantification is entered, it is then possible to fit the quantification as a linear combination of phases. A simple example would be to fit a pyroxene composition against a linear combination of enstatite, ferrosilite, and wollastonite. Another example would be to improve the iron quantification in an olivine containing nanophase troilite. One could fit forsterite-fayalite$\mathrm{FeS}$ and obtain an improved Fo\#. It is possible to fit any phases you wish, including phases defined by the user, e.g. "chondritic solids" which has a solar abundance of every element except oxygen which is computed by stoichiometry.

However, it is important to fit fewer phases than there are elements, and avoid fitting phases with overlapping elements. For example, a spectrum containing $\mathrm{Mg}, \mathrm{Ca}, \mathrm{Fe}, \mathrm{Si}$ and $\mathrm{O}$ cannot be fit against a series: forsterite, fayalite, monticellite, enstatite and ferrosilite. Such a fit would always produce "perfect" results but would produce $\mathrm{Mg \# s}$ which were not reliable.

For more sophisticated analyses, python scripts (named phase analyses) can be run on the quantification to extract information specific to an analytic workflow. These can be very simple as few as four lines of code - or they can be very complex. For example, iron sulfides have a number of superlattices which are a function of the cation/S ratio. An analysis script named "sulfide" calculates the cation/S ratio and then suggests the superlattice structure along with the reference on which the suggestion is based [3]. An analysis script named "spinel" tests for the correct stoichiometry and warns in case errors are found. If not, it computes octahedral and tetrahedral occupancies and charges [4]. This is both more robust and more flexible than the spreadsheet approach commonly used in the geological sciences.

Since the scripts are in a generic language (python) they can be used to produce production quality plots, compare values against databases, compare against web sites, and do any sort of analysis the science requires. 
Since both the input data and the output analyses are saved by the program, reproducibility is aided since you can see exactly what was done at a later date.

The software has been in use internally for two years and is robust for the workflow of the authors. In order to make the software broadly accessible and extensible by the community, we have made the software open source and hosted it on Github [5]. A short video showing the software in use is also hosted. The software is dependent on numpy[7], matplotlib[8] and wxPython[9]. We hope you will find Stoichiometry Fitter useful, enjoyable to use, and that it will increase your productivity. [10]

References:

[1] B.L. Henke, E.M. Gullikson, and J.C. Davis., At. Data Nucl. Data Tables 54, 2, (1993), p. 181.

[2] http://henke.lbl.gov/optical_constants/

[3] H. Nakazawa, and N. Morimoto, Materials Research Bulletin 6(5) (1971), 345.

[4] N. Morimoto, J. Fabries, A. K. Ferguson, et al. Am. Min. 73 (1988), p. 1123.

[5] https://github.com/ZGainsforth/StoichiometryFitter

[7] Stéfan van der Walt, S. Chris Colbert and Gaël Varoquaux. Comput. Sci. Eng. 13 (2011), p. 22

[8] John D. Hunter. Comput. Sci. Eng. 9 (2007), p. 90

[9] http://www.wxpython.org

[10] Work at the Molecular Foundry was supported by the Office of Science, Office of Basic Energy Sciences, of the U.S. Department of Energy under Contract No. DE-AC02-
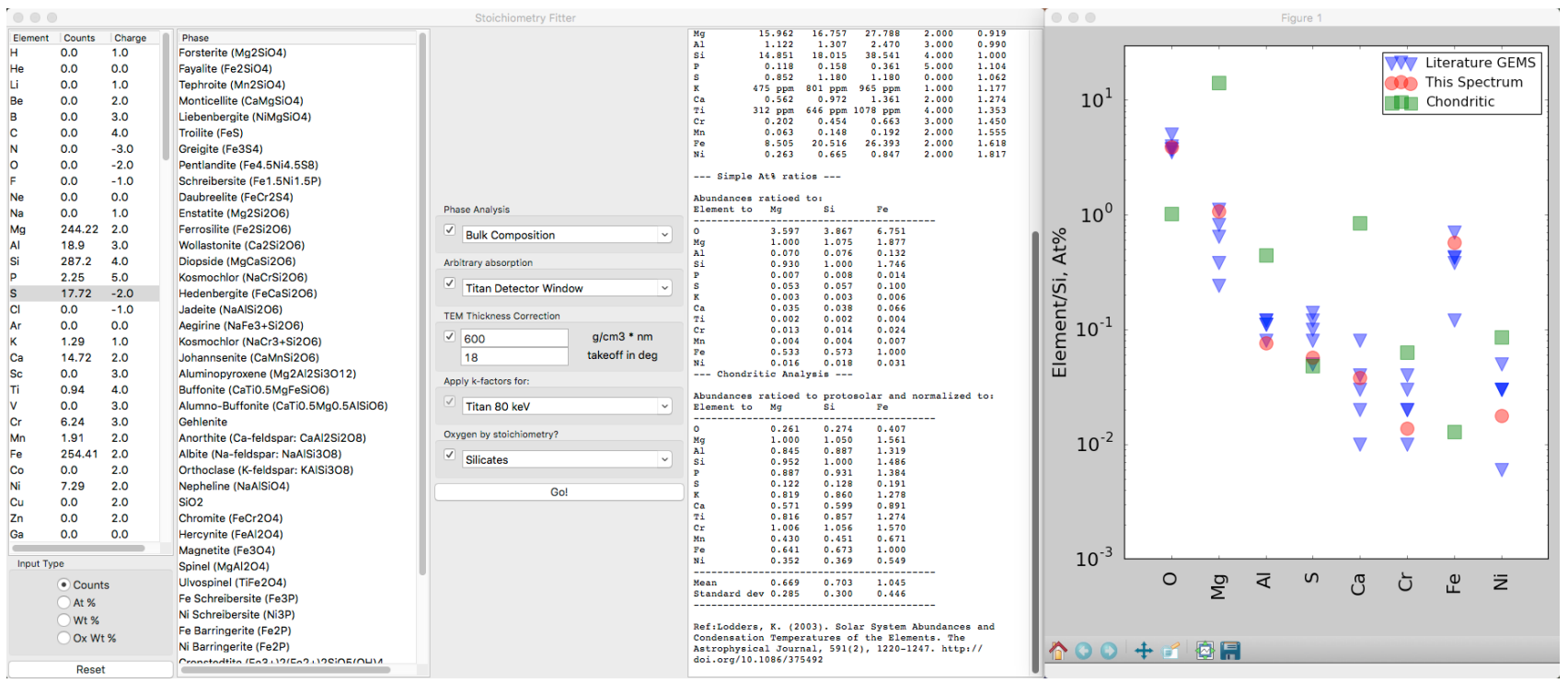

$05 \mathrm{CH} 11231$.

Figure 1. A screen shot of an analysis involving TEM k-factors, thickness and detector corrections, and a phase analysis. 